\title{
Concurrent Failure of Active and Redundant Ventricular Epicardial
} Electrodes in Children

\author{
GERALD A. SERWER, M.D., MACDONALD DICK II, M.D., KAREN UZARK, R.N., Ph.D., \\ WILLIAM A. SCOTT, M.D., and EDWARD L. BOVE, M.D. \\ From the Department of Pediatrics and Thoracic Surgery, C.S. Mott Children's Hospital, University of Michigan Medical Center, \\ Ann Arbor, Michigan
}

\begin{abstract}
Implanting an unused or redundant epicardial ventricular pacing electrode in children at the time of initial electrode placement for later use is a common practice. To investigate the ultimate utilization of this redundant electrode, the records of 38 children who had undergone implantation of 47 redundant epicardial electrodes between 1974 and 1987 were reviewed. As the primary electrode remains functional, 12 electrodes have not been invasively tested or used; 11 were in nine children lost to follow-up. The remaining 24 redundant electrodes implanted in 16 children, (ages 1 day to 18 years, median 5 years, at implantation) together with the utilized electrode were evaluated at the time of pacing system revision. At implant, all electrodes had thresholds $<1.4$ volts at a pulse duration of 0.5 msec. At follow-up 0.2 to 11.7 years (mean 3.7 years) after implant, an electrode was considered functional if the threshold at $0.5 \mathrm{msec}$
\end{abstract}

\section{Introduction}

The epicardial pacing electrode remains the most commonly used pacing electrode in children. ${ }^{1,2}$ As open heart surgical procedures are done in smaller children, the need for permanent pacing in smaller children has increased. Even though we are now implanting transvenous endocardial electrodes in smaller children, there is a limit below which it is not yet possible to go. In addition, there are patients in whom endocardial placement is impossible or contraindicated. Patients following

Address for reprints: Gerald A. Serwer, M.D., Room F1615, Box 0204, C.S. Mott Children's Hospital, University of Michigan Medical Center, Ann Arbor, MI 48 109-0204. pulse duration was $<2.5$ volts, and 14 of 24 redundant electrodes were nonfunctional. Of the ten functional redundant electrodes, the active electrode was functional in seven cases. In only three of 24 cases was the redundant electrode functional when the active electrode was nonfunctional. In all cases, when the active electrode exhibited exit block, the redundant electrode also exhibited exit block. Two of five redundant electrodes paired with a fractured active electrode were fractured. Two other redundant electrodes were fractured when the active electrode was functional. The state of the redundant and the active electrode was the same in $80 \%$ of the cases, $P<0.03$. Of the 36 redundant electrodes implanted for which followup data is available, 33 had not been used by the end of the study period. ( $\mathrm{J}$ Interven Cardiol 1989:2:1)

Fontan's procedure cannot have endocardial electrodes placed and patients following Mustard or Sennings's procedure who have SVC obstruction cannot have endocardial electrodes placed. There are still many programs in which epicardial electrode placement is far more common than endocardial placement such that the point made in our article is by no means moot.

The majority of epicardial pacing systems utilize a unipolar generator requiring only one active electrode. ${ }^{3,4}$ However, as a surgical thoracotomy for electrode placement is needed, a nonused or redundant pacing electrode is often placed in anticipation that should the active electrode malfunction, the redundant electrode will serve as a replacement averting the need for a subsequent thoracotomy. Our practice has been to implant 
two electrodes, utilizing one as the active electrode and capping the second, leaving this redundant electrode to serve as an easily accessible backup should the original electrode fail. However, whether such a practice does defer the need for a subsequent thoracotomy is unknown. The hypothesis that implanting such a redundant electrode does postpone the need for future thoracotomy is predicated on the assumption that electrode failure rates are different for simultaneously placed active and redundant electrodes. We evaluated this hypothesis by assessing the ultimate fate of both the active and redundant electrodes and the number of instances in which the redundant epicardial electrode was used.

\section{Materials and Methods}

Patient Population. Records of all children undergoing pacemaker system implantation or revision between January 1, 1974, and December 31, 1987, at C.S. Mott Children's Hospital at the University of Michigan were reviewed with 38 children identified who had undergone placement of 47 redundant ventricular epicardial pacing electrodes and 47 simultaneously implanted active ventricular electrodes of the same type as the redundant electrode. Nine of these children who had undergone placement of 11 redundant electrodes were not available for follow-up data (five died and four moved); 12 redundant electrodes have not been tested as the original active electrode and generator continue to function. The remaining 24 redundant electrodes implanted in 16 children comprise the study group.

The patient ages at electrode implantation were 1 day to 18 years, median 5 years (Table I). Seven children had required pacemaker implantation for surgically induced heart block, four for congenital heart block with either congestive heart failure or syncopal episodes, and five for treatment of sick sinus syndrome-two of these associated with a cardiomyopathy and three following Mustard's procedure for correction of transposition of the great arteries. Of the 16 children, 11 had congenital structural cardiac disease and two had a cardiomyopathy.

Of the 24 redundant electrodes implanted, four were implanted at the time of open heart surgery
Table I. Patient Characteristics

\begin{tabular}{rcll}
\hline Patient & $\begin{array}{c}\text { Age at Initial } \\
\text { Implant } \\
\text { (Yrs) }\end{array}$ & Diagnosis & $\begin{array}{c}\text { Indication for } \\
\text { Pacing }\end{array}$ \\
\hline 1 & 3.8 & VSD & Surg HB \\
2 & 13.9 & dTGA & SSS \\
3 & 3.9 & ASD & Cong HB \\
4 & 1.9 & dTGA & Surg HB \\
5 & 2.2 & AVSD & Surg HB \\
6 & 17.4 & dTGA & SSS \\
7 & 2.8 & Nl & Cong HB \\
8 & 6.3 & VSD & Surg HB \\
9 & 7.3 & dTGA & Surg HB \\
10 & 7.8 & dTGA & SSS \\
11 & 0.01 & Nl & Cong HB \\
12 & 13.0 & TA & Surg HB \\
13 & 0.01 & NI & Cong HB \\
14 & 7.3 & VSD & Surg HB \\
15 & 1.2 & CM & SSS \\
16 & 1.4 & CM & SSS \\
\hline
\end{tabular}

VSD $=$ Ventricular Septal defect; dTGA = d-Transposition of the great arteries; ASD = secundum atrial septal defect; AVSD $=$ atrioventricular septal defect; $\mathrm{NI}=$ no structural disease; TA = tricuspid atresia; $\mathrm{CM}=$ cardiomyopathy; Surg $\mathrm{HB}=$ surgically induced heart block; SSS = sick sinus syndrome; Cong HB $=$ congenital heart block.

and two were implanted at a separate surgical procedure 1 week following open heart surgery. The remainder were implanted at a separate surgical procedure far removed from any other cardiac surgical procedure. All electrodes were implanted via a thoracotomy and tunneled subcutaneously to an abdominal generator pocket. All electrodes except those implanted in children with transposition of the great arteries were implanted on the left ventricle. The active and redundant electrode were implanted on the same ventricle in all cases. The surgeons endeavored in all cases to implant the electrodes as far apart as was possible, but this distance varied greatly dependent upon the degree of the myocardium that was exposed and where the surgeon felt he could find viable myocardium in which to implant the electrode. None of the redundant electrodes were utilized initially as all children had implantation of a unipolar generator.

Electrode Characteristics. Of these 24 electrodes ten were Medtronic Inc. Model 6917 or 6917 A; seven were Model 5815; four were Model 4951 (Medtronic, Inc., Minneapolis, MN, USA); two were CPI Model 4313 (CPI, St. Paul, MN, USA); 


\section{REDUNDANT VENTRICULAR EPICARDIAL ELECTRODE FAILURE}

and one was a Coratomic Model L-30. At the time of initial implantation, all electrodes had their thresholds determined and had voltage thresholds $<1.4$ volts at a pulse duration of $0.5 \mathrm{msec}$. Electrode impedance ranged from 350 to $710 \mathrm{ohms}$. Electrode age at follow-up evaluation was 0.2 to 11.7 years with a median age of 3.5 years and a mean age of 3.7 years, Table II.

Protocol. At the time of pacing system revision, both the currently utilized and the redundant electrode were evaluated. Threshold measurements were performed on both electrodes with either a Medtronic model 5300 or 5311 pacing system analyzer (Medtronic, Inc., Minneapolis, MN, USA) using the electrode and a grounding plate in the generator pocket. The minimum voltage needed to produce $100 \%$ capture was determined at a pulse duration of $0.5 \mathrm{msec}$. Electrode impedance and the $\mathbf{R}$ wave amplitude generated by a spontaneous beat were measured.
Definitions. An electrode was considered to be nonfunctional if its threshold was greater than 2.5 volts at a pulse duration of $0.5 \mathrm{msec}$. This threshold was chosen based upon pacemaker manufacturers recommendations. This is a consequence of the high likelihood of loss of capture even for generators capable of 8.0 volt outputs when thresholds exceed this value. If an electrode was classified as nonfunctional, it was then further classified as either having failed due to exit block or fracture. Exit block was defined as a failed electrode with impedance $>300$ ohms but which still would capture at a very high output. None of the electrodes showing exit block were even close to our limit for acceptability. Most exhibited voltage thresholds greater than 4 volts at $0.5 \mathrm{msec}$ with significant elevation of the measured impedance. A fractured electrode was defined as a failed electrode with either an impedance $<300 \mathrm{ohms}$, implying insulation fracture or tip erosion into the ventricular

Table II. Electrode Data

\begin{tabular}{|c|c|c|c|c|}
\hline $\begin{array}{l}\text { Electrode } \\
\text { Model }\end{array}$ & $\begin{array}{c}\text { Pt. Age at } \\
\text { Implant (YTs) }\end{array}$ & $\begin{array}{c}\text { Electrode Age } \\
\text { at Follow-up } \\
\text { (Yrs) }\end{array}$ & $\begin{array}{l}\text { Redundant } \\
\text { Electrode } \\
\text { Status }\end{array}$ & $\begin{array}{c}\text { Active } \\
\text { Electrode } \\
\text { Status }\end{array}$ \\
\hline Cor L-30 & 6.3 & 9.8 & $\mathrm{~EB}$ & EB \\
\hline CPI 4313 & 13.1 & 4.8 & $\mathrm{G}$ & $G$ \\
\hline CPI 4313 & 3.3 & 4.4 & Frac & $G$ \\
\hline Med 4951 & 1.4 & 2.6 & $\mathrm{~EB}$ & EB \\
\hline Med 4951 & 11.7 & 0.2 & $\mathrm{~EB}$ & EB \\
\hline Med 4951 & 12.1 & 0.5 & EB & $\mathrm{EB}$ \\
\hline Med 4951 & 4.0 & 0.2 & Frac & Frac \\
\hline Med 5815 & 1.9 & 11.7 & Frac & $\mathrm{G}$ \\
\hline Med 5815 & 10.2 & 5.1 & G & Frac \\
\hline Med 5815 & 13.9 & 2.8 & Frac & Frac \\
\hline Med 5815 & 2.8 & 5.7 & G & Frac \\
\hline Med 5815 & 3.9 & 2.0 & EB & EB \\
\hline Med 5815 & 5.9 & 0.3 & $G$ & Frac \\
\hline Med 5815 & 7.3 & 1.8 & $\mathrm{~EB}$ & $\mathrm{~EB}$ \\
\hline Med 6917 & 0.03 & 5.4 & $\mathbf{G}$ & $\mathrm{G}$ \\
\hline Med 6917 & 1.4 & 4.0 & $\mathbf{G}$ & $\mathrm{G}$ \\
\hline Med 6917 & 13.0 & 0.2 & EB & $\mathrm{EB}$ \\
\hline Med 6917 & 13.0 & 6.7 & $\mathrm{G}$ & $\mathrm{G}$ \\
\hline Med 6917 & 16.7 & 0.3 & $\mathrm{G}$ & $\mathrm{G}$ \\
\hline Med 6917 & 17.4 & 4.3 & $\mathrm{G}$ & $\mathrm{G}$ \\
\hline Med 6917 & 2.1 & 0.3 & $\mathrm{~EB}$ & EB \\
\hline Med 6917 & 2.4 & 3.0 & $\mathrm{G}$ & $\mathrm{G}$ \\
\hline Med 6917 & 6.0 & 5.7 & EB & EB \\
\hline Med 6917 & 9.2 & 3.8 & $\mathrm{~EB}$ & EB \\
\hline
\end{tabular}

Pt $=$ patient; Med = Medtronic, Inc.; Cor = Coratomic, Inc.; CPI = Cardiac Pacemakers, Inc.; EB = electrode exhibiting exit block; G $=$ functioning electrode; $F r a c=$ fractured electrode. 
cavity, or inability to pace the heart using the electrode at any output up to an amplitude of 10 volts and a pulse width of $2 \mathrm{msec}$ implying conductor fracture.

\section{Results}

Of the 24 redundant electrodes evaluated, 14 or $58 \%$ were nonfunctional at the time of evaluation (Table II). In addition, 15 of the 24 active electrodes, or $63 \%$, were nonfunctional. The remaining ten redundant electrodes were functional, but seven of the ten active electrodes were also functional. In only three cases was the redundant electrode functional when the active electrode was nonfunctional, thus providing a usable electrode without the need for further electrode implantation. Conversely, only two active electrodes were functional when the redundant electrode was nonfunctional. Therefore, there were five of 24 electrode pairs that differed in status (20\%). The state of the active electrode was highly correlative with the state of the redundant electrode, $\mathrm{P}<0.03, \mathrm{X}^{2}$ $=5.53$.

Of the 14 failed redundant electrodes, 10 exhibited exit block. The paired active electrode for all 10 also exhibited exit block. All such electrodes had voltage thresholds well above 2.5 volts. No case was found in which exit block occurred in either the active or redundant electrode, but not in the other. Four redundant electrodes were fractured. Two had associated active electrodes that were also fractured while two had an associated active electrode that was functional. Three active electrodes were fractured when the redundant electrode was functional. The mean age of the redundant electrodes exhibiting exit block and fractures was not significantly different $(3.06 \pm 3.0$ [SD] vs $4.87 \pm 5.1$ years). The electrodes exhibiting exit block were equally divided between all electrode types. No Medtronic 6917 electrode was found to be fractured, whereas all other types exhibited electrode fracture.

Of the 24 redundant electrodes evaluated, 21 or $88 \%$ were never utilized during the follow-up period. Of the 36 total redundant electrodes for which follow-up data was available, 33 had not been used by the end of the study period.

\section{Discussion}

This study clearly demonstrates that redundant epicardial electrodes are rarely utilized and only in a rare instance do they obviate the need for a repeat thoracotomy for electrode implantation as the state of the redundant electrode is highly correlative with the state of the active electrode. Both are equally likely in a given patient to develop exit block or fracture.

Electrode redundancy has been proposed as an advantage of bipolar pacing. ${ }^{5}$ Holmes et al. proposed that the redundancy of a bipolar system permitted changing from bipolar to unipolar or reversing polarity to maximize electrode performance. In their series, 34 of 248 patients had improved electrode thresholds by either changing to a unipolar system or changing polarity. Of these 248 patients only eight had epicardial electrodes. Yet, electrode thresholds were initiallty acceptable in 16 of the 34 patients, and while changing polarity or converting to a unipolar system improved thresholds, new electrodes would not have been needed. In only 18 of 248 patients (7.2\%) did redundancy prevent further electrode implantation. Thus, in our experience we have not found this practice useful.

Another form of pacing redundancy has been proposed by Morse et al. ${ }^{6}$ They reported a case in which two ventricular epicardial electrodes were implanted and attached to a dual chamber generator with the AV interval programmed to a minimal setting and the mode set to DDD. In this instance the "ventricular" channel paces during ventricular refractoriness with no effect. Should the "atrial" channel fail to capture, the "ventricular" channel serves as a backup. This approach requires a more sophisticated pulse generator with a higher current drain and thus a decreased longevity and still depends upon at least one functioning electrode. Our data suggest that both the redundant and active electrodes are most likely to fail together or both function normally.

Both electrode longevity and the ease with which a new electrode can be inserted influence the desirability of electrode redundancy. Longer electrode survival reduces the need for electrode redundancy. In an early series by Simon et al. ${ }^{7}$ epicardial electrode longevity was low with a $50 \%$ failure rate by 62 months. A more recent series by 


\section{REDUNDANT VENTRICULAR EPICARDIAL ELECTRODE FAILURE}

Serwer et al. ${ }^{8}$ demonstrated longer epicardial electrode survival with a $50 \%$ failure rate not occurring until 100 months. In addition, exit block has been shown to be the single most common reason for epicardial electrode failure. ${ }^{8,9}$ Our work confirms this finding and also shows that exit block in the active electrode is highly associated with exit block in the redundant electrode.

Another factor influencing the use of electrode redundancy is the increased use of endocardial electrodes in children. Our current practice is to implant endocardial pacing systems in children $>15 \mathrm{~kg}$ in weight in whom there are no contraindications such as superior vena caval obstruction, right to left shunting, and/or preexisting pulmonary hypertension. ${ }^{2,10,11}$ When pacing is needed in small children, epicardial pacing systems are employed. By the time pacing system revision is needed, conversion to an endocardial system is usually possible. Therefore, a redundant epicardial electrode is of no utility even if it is functional. Only in those patients for whom endocardial electrode placement is contraindicated or those developing quite early electrode failure would a redundant epicardial electrode be of any potential benefit. Prior work shows generator longevity $(50 \%$ survival-63 months) to be less than electrode longevity (50\% survival-100 months) and to be the single largest indication for pacing system revision. ${ }^{4,8}$ Thus, conversion to an endocardial pacing system at the time of generator failure may be performed prior to initial electrode failure.

Not only are there no advantages to placement to a redundant electrode, there may, in fact, be disadvantages. The second electrode placement increases the potential for myocardial damage with a second site of injury and theoretically this could adversely affect the threshold of the first electrode. Also, the placement of additional foreign material increases the risk and consequences of infection.

With advances in epicardial electrode design and the concomitant increased use of the endocardial electrode in children, the usefulness of the redundant epicardial electrode has decreased to the point we feel it is no longer indicated in the absence of contraindications to eventual endocardial pacing.

\section{References}

1. Wampler DG, Shannon C, Burns GV, et al. Cardiac pacing in children and young adults. Am J Dis Child 1983; 137:1098-1100.

2. Gillette PC, Shannon C. Cardiac pacing in children. Cardiovasc Clin 1983; 14:209-226.

3. Oldershaw PJ, Sutton MG, Ward D, et al. Ten-year experience of 359 epicardial pacemaker systems: Complications and results. Clin Cardiol 1982; 5:515-519.

4. Serwer GA, Mericle JM. Evaluation of pacemaker pulse generator and patient longevity in patients aged 1 day to 20 years. Am J Cardiol 1987; 59:824-827.

5. Holmes DR Jr, Gersh BJ, Shub C, et al. The value of redundancy in chronic bipolar pacemaker electrode systems. PACE 1980; 3:436-439.

6. Morse DP, Tarjan $\mathrm{P}, \mathrm{McNicholas} \mathrm{KW}$, et al. Redundant pacing: Case report and review. PACE 1984; 7:296-300.

7. Simon AB, Dick M II, Stern AM, et al. Ventricular pacing in children. PACE 1982; 5:836-844.

8. Serwer GA, Mericle JM, Armstrong BE. Epicardial ventricular pacemaker longevity in children. Am J Cardiol $1988 ; 61: 104-106$.

9. Williams WG, Izukawa T, Olley PM, et al. Pemanent cardiac pacing in infants and children. PACE 1978; $1: 439-447$

10. Gillette PC, Shannon C, Blair H, et al. Transvenous pacing in pediatric patients. Am Heart J 1983; 105:843-847.

11. Hayes DL, Holmes DR Jr, Maloney JD, et al. Permanent endocardial pacing in pediatric patients. $\mathrm{J}$ Thorac Cardiovasc Surg 1983; 85:618-624. 